AN. MED. InTERNA (Madrid) Vol. 19, N. $^{\circ} 9$, pp. $457-459,2002$

\title{
Linfoma T primario intestinal: a propósito de dos casos con revisión de la literatura
}

\author{
R, MOLINA VILLAVERDE, A. JIMÉNEZ GORDO, J. L. LÓPEZ GONZÁLEZ, \\ A. REDONDO SÁNCHEZ, M. ANDREU RODRÍGUEZ, M. GONZÁLEZ BARÓN
}

\author{
Servicio de Oncología Médica. Hospital La Paz. Madrid
}

\author{
PRIMARY INTESTINAL T LYMPHOMA: A REPORT OF TWO CASES \\ WITH A REVIEW OF THE LITERATURE
}

\begin{abstract}
RESUMEN
Los linfomas del tracto gastrointestinal son el tipo más frecuente de linfomas extraganglionares y de éstos el 15-20\% son de localización intestinal. Pueden ser B o T. Los linfomas tipo T son menos comunes y pueden estar o no asociados a enteropatía. Suelen aparecer en adultos en forma de dolor abdominal a menudo asociado a perforación intestinal. El curso es agresivo. El mayor problema es hacer el diagnóstico diferencial con las úlceras benignas. Se presentan dos casos de diferentes características y evolución y que permiten realizar una revisión de las características clinicopatológicas de esta rara entidad.
\end{abstract}

PALABRAS CLAVE:Linfoma T primario intestinal.

\begin{abstract}
Lymphomas of the gastrointestinal tract are the most common type of primary extranodal lymphomas and about 15-20\% of these are primary intestinal lymphomas. They may be or B or T-cell. Intestinal Tcell lymphomas are much less common and they can be enteropathyassociated. This disease occurs in adults with abdominal pain often asso ciated with intestinal perforation. The course is aggressive. The major problem is to distinguish this disease from a benign ulcer. Two cases with differents clinical and pathologic features are reported with a review in the literature of this uncommon entity.
\end{abstract}

Molina Villaverde R, Jiménez Gordo A, López González JL, Redondo Sánchez A, Andreu Rodríguez M, Manuel González Barón. Linfoma T primario intestinal: a propósito de dos casos con revisión de la literatura. An Med Interna (Madrid) 2002; 19: 457-459.

\section{INTRODUCCIÓN}

Los linfomas del tracto gastrointestinal son el tipo de linfomas primarios extranodales más frecuentes y constituyen entre un 5 y un $10 \%$ de todos los linfomas no Hodgkin (1) y entre un 1 y un $4 \%$ de todos los tumores malignos del tracto digestivo $(2,3)$. Los linfomas intestinales representan un 15 a un $20 \%$ de todos los del tubo gastrointestinal (4). La clasificación de Isaacson y cols es la más utillizada para esta enfermedad (Tabla I) $(5,6)$ y según esta pueden ser B o T. Los de tipo $\mathrm{T}$ son menos comunes y pueden estar o no asociados a enteropatía ( esto último es lo menos habitual) $(7,8)$. En la mayoría de los casos los linfomas $\mathrm{T}$ no asociados a enteropatía son de alto grado de malignidad $(9,10)$.

Se presentan dos casos de linfomas $\mathrm{T}$ intestinales primarios con distinta evolución y pronóstico, además de revisar las características clinicopatológicas de esta rara entidad.

\section{CASOS APORTADOS}

Caso 1: Paciente varón de 35 años sin antecedentes de interés que acude al Servicio de Urgencias por dolor abdominal de intensidad creciente con signos de irritación peritoneal en la exploración por lo que fue intervenido objetivándose una tumoración yeyunal a unos 20 centímetros que se extirpó realizándose una anastomosis yeyunoyeyunal. Durante el acto quirúrgico se apreciaron lesiones de aspecto neoplásico que infiltraban difusamente el mesentereo. La anatomía patológica mostró la existencia de una proliferación tumoral de células de núcleos grandes con nucleolo prominente y escaso citoplasma que crecían en sábana infiltrando tanto la lámina propia como la submucosa llegando hasta serosa. Las células correspondían a linfocitos $\mathrm{T}$ existiendo muy pocos linfocitos $\mathrm{B}$ acompañantes. El estudio de extensión fue negativo (incluida la tomografía axial computerizada y la biopsia de médula ósea).

Fue remitido al Servicio de Oncología para tratamiento adyuvante. Se administraron 6 ciclos de quimioterapia con Ciclofosfamida $\left(750 \mathrm{mg} / \mathrm{m}^{2}\right)$, Adriamicina $\left(40 \mathrm{mg} / \mathrm{m}^{2}\right)$, Vincristina $\left(1,4 \mathrm{mg} / \mathrm{m}^{2}\right)$ y

Trabajo aceptado: 9 de marzo de 2001. 


\section{TABLA ।}

\section{CLASIFICACIÓN DE LOS LINFOM AS PRIM ARIOS} INTESTINALES

\author{
Linfomas de células $B$ \\ Tipo M ALT: \\ Bajo grado. \\ Alto grado con o sin componente de bajo grado aso- \\ ciado. \\ Enfermedad inmunoproliferativa de intestino delgado- \\ de bajo grado o de alto grado con o sin componenete \\ de bajo grado. \\ Poliposis linfomatosa. \\ Linfoma de Burkitt y Burkitt-like. \\ O tros tipos de linfomas de bajo o de alto grado corres- \\ pondientes a equivalentes linfoides ganglionares. \\ Linfomas de células T \\ Linfoma T asociado a enfermedad celíaca o enteropatía. \\ O tros linfomas T no asociados a enteropatía.
}

Prednisona $\left(100 \mathrm{mg} / \mathrm{m}^{2}\right)$. El paciente ha seguido revisiones sin objetivarse recidiva.

Caso 2: Paciente varón de 62 años con antecedentes de tabaquismo y bebedor moderado de alcohol que ingresa en el Servicio de Medicina Interna por astenia, anorexia y fiebre con un cuadro de diarrea de dos meses de evolución. Se realizó tránsito intestinal en el que aparecía un engrosamiento de los pliegues duodenales con sospecha inicial de enfermedad celíaca del aduto de larga evolución o de un posible linfoma. En la ecografía se objetivó una hepatoesplenomegalia con hipertensión portal y una imagen nodular en lóbulo hepático derecho con punción- aspiración que mostraba una intensa linfocitosis sin poder establecer claramente si se trataba de un proceso linfoproliferativo. Se practicó resección intestinal y la anatomía patológica fue de linfoma no Hodgkin tipo T. No se pudo continuar el estudio ni el tratamiento puesto que el paciente falleció por una complicación respiratoria durante el postoperatorio.

\section{DISCUSIÓN}

Los linfomas primarios del intestino delgado tienen una presentación clínica, un pronóstico y unos rasgos patológicos completamente diferentes a los de otras localizaciones del tracto digestivo. En conjunto son el doble de frecuentes en el sexo masculino con respecto al femenino, con una media de edad de 50-60 años (11). Esta enfermedad se clasifica actualmente según el sistema propuesto por Isaacson y cols. El linfoma $\mathrm{T}$ intestinal es un tipo raro de linfoma extraganglionar derivado de las células $\mathrm{T}$ intraepiteliales que originalmente se denominó histiocitosis maligna intestinal $(12,13)$. Puede estar asociado a enteropatía o no. Como en el resto de los linfomas la edad media del diagnóstico es de 60 años y es tres veces más frecuente en varones. En un estudio con 31 pacientes con linfomas intestinales primarios se vio que los que tenían linfomas $\mathrm{T}$ eran generalmente hombres jóvenes con una edad media de 34 años (14).

La clínica suele ser de dolor abdominal que con frecuencia se asocia a perforación u obstrucción intestinal $(15,16)$. Se localiza preferentemente en el yeyuno en forma de lesiones ulcero- sas macroscópicas circunferenciales sin la formación, a diferencia de los linfomas de tipo B, de grandes masa tumorales. En un $70 \%$ de pacientes la enfermedad es multifocal. A veces puede acompañarse de un síndrome general con astenia y pérdida de peso (17). A veces la presentación es como la de una isquemia intestinal pero que afecta preferentemente a pacientes de menor edad y con una evolución muy ominosa (18).

Microscópicamente aparecen cúmulos de células linfoides de pequeño tamaño que forman agrupaciones intraepiteliales. En los tumores ulcerados, el infiltrado celular inflamatorio es muy intenso e incluso puede hacer difícil el diagnóstico por la dificultad de diferenciar las células tumorales. Actualmente se considera que la mayoría, pero no todos los casos de linfoma $\mathrm{T}$ se asocian a enteropatía. Los que no están asociados son de alta malignidad y tiene muy mal pronóstico (19).

Se ha determinado una supervivencia para los linfomas primarios $\mathrm{T}$ a los cinco años del 13 al $25 \%$, debido a una alta tasa de mortalidad perioperatoria $(14,18)$. Los factores pronósticos valorados son varios y la importancia de unos u otros dependen de los estudios realizados (Tabla II) (20-22). El factor más claro de todos parece ser el estadio tumoral. La enfermedad limitada tiene una tasa de supervivencia a los dos años del $80 \%$ si es estadio I y del $60 \%$ si es II. La supervivencia también esta en relación con la posibilidad de resección com-

\section{TABLA ॥}

\section{FACTO RES PRO NÓ STICOS EN EL LINFOM A INTESTINAL} PRIM ARIO

\author{
Estadio tumoral. \\ Edad de presentación (peor en edades extremas). \\ Modo de presentación clínica (peor si perforación intestinal). \\ Estado general del paciente (performance status). \\ Masa tumoral mayor de $7 \mathrm{~cm}$. \\ Histología y grado de malignidad. \\ Invasión parietal. \\ Presencia de inmunosupresión asociada. \\ M ultifocalidad del tumor.
}

pleta tumoral aunque esta no es posible si la enfermedad está avanzada (estadios III o IV).

Los dos casos presentados tienen características diferentes y se dieron a distintas edades aunque ambos en varones. La forma de presentación en el primer caso fue la de un abdomen agudo que requirió intervención urgente que probablemente determinó el pronóstico futuro del paciente ya que pudo ser completamente resecado. En el otro caso la sintomatología inicial fue insidiosa y permitió un estudio de extensión inicial y el planteamiento de otras patologías en el diagnóstico diferencial, sobre todo con las lesiones compatibles con un síndrome de malabsorción. En este paciente, sin embargo, el pronóstico fue peor debido a una complicación perioperatoria.

El mayor problema de esta entidad es que al no asociarse a masa abdominal dificulta su distinción de otras enfermedades benignas o bien malabsortivas o bien que producen ulceraciones. No obstante, hay que incluirla en el diagnóstico diferencial ante una sintomatología de este tipo. 


\section{Bibliografía}

1. Zinzani PL, Magnagnoli M, Pagliani G, et al. Primary intestinal lymphoma: clinical and terapeutic features of 32 patients. Haematologica 1997; 82: 3: 305-8

2. Bush RS: Primary lymphomas of the gastrointestinal tract. JAMA 1974; 228: 1291-4.

3. Freeman C, Berg JW, Cutler SJ. Ocurrence and prognosis of extranodal lymphoma. Cancer 1972; 29: 252-60.

4. Crump M, Gosporadowiez M, Sepherd FA. Lymphoma of the gastrointestinal tract. Sem Oncol 1999; 26: 324-37.

5. Isaacson P. Gastrointestinal lymphoma. Hum Pathol 1994; 25: 1020-9.

6. Lipford EH, Margolick JB, Longo DL, Fauci AS, Jaffe ES. Angiocentric inmunoproliferative lesions: a clinicopathologic spectrum of postthymic T- cell proliferations. Blood 1988; 72: 1674-81.

7. Remacha B, Palau A, Velicia R, Caro A, Ripollés V. Primary intestinal T lymphoma. Rev Esp Enferm Dig 1998; 90 (3): 183-90.

8. Lavergne A, Brocheriou I, Delfou MH, et al. Primary intestinal gammadelta T-cell lymphoma with evidence of Epstein Barr virus. Histopathol 1998; 32 (3): 271-6.

9. Grogan TM, Fielder K, Rangel C, et al. Peripheral T- cell lymphoma: aggressive disease with heterogeneous inmunotypes. Am J Clin Pathol 1985; 83: 279-87.

10. Salter DM, Krajewski AS, Dewar AE. Inmunophenotype analysis of malignant histiocytosis of the intestine. J Clin Pathol 1986; 39: 815.

11. Dragosics B, Bauer P, Radaszkiewicz T. Primary gastrointestinal nonHodgkin's lymphomas. A retrospective clinicopathologic study of 150 cases. Cancer 1985; 55: 1060-73.

12. Schmitt GA, Daum S, Humel M, Zemlin M, Stein H, Riecken EO. Presence of clonal $\mathrm{T}$ - cell receptor gene rearragements provides evidence of widespread intramucosal intestinal T- cell lymphoma. Z- Gastroenterol 1996; 34 (10): 680-5.
13. Isaacson P, Spencer J, Connolly C. Malignant histiocytosis of the intes tine: a T- cell lymphoma. Lancet 1985; 2: 688-91.

14. Li G, Ouyang Q, Liu K, Wang Y, Yang X. Primary non-Hodgkin's lymphoma of the intestine: a morphological, inmunohistochemical and clinical study of 31 chinese cases. Histopathology 1994; 25: 113-21.

15. Shipp M, Mauch P, Harris N. Non- Hodgkin's lymphomas. En: DeVita V editors. Principles and Practice of Oncology . Philadelpia. LippincottRaven Publishers, 1997. p. 1131-2283.

16. Al- Mondhiry H. Primary lymphomas of the small intestine: East- West contrast. Am J Hematol 1986; 22: 89-105.

17. Chott A, Dragosics B, Radaszkiewicz T. Peripheral T- cell lymphomas of the intestine. Am J Pathol 1992; 141: 1361-71.

18. Shepherd NA, Hall PA, Williams G, et al. Primary malignant lymphoma of the large intestine complicating chronic inflammatory bowel disease. Histopathology 1989; 15: 325-37.

19. Grogan TM, Fielder K, Rangel C, et al. Peripheral T- cell lymphoma: agressive disease with heterogeneous inmunotypes. Am J Clin Pathol 1985; 83: 279-87.

20. Aozasa K, Ueda T, Kurata A, et al. Prognostic calue of histologic and clinical factors in 56 patients with gastrointestinal lymphomas. Cancer 1988; 61: 309-15.

21. Hermann R, Panahon AM, Barcos MP, Walsh D, Stutzman L. Gastrointestinal involvement in non- Hodgkin's lymphoma. Cancer 1980; 46: 215-22.

22. Espinosa E, Zamora P, González Barón M. Factores pronósticos y predicción de la respuesta en los linfomas de alto grado. En: González Barón M and et editors. Fractores pronósticos en Oncología. Madrid. Interamericana McGraw Hill 1994. p. 367-78.

23. dÁmore F, Brincker H, Gronback K, Thorling K, Pedersen M, Jensen MK, et al. Non- Hodgkkin's lymphoma of the gastrointestinal tract: A population based analysis of incidence, geographic distribution, clinicopathologic presentation features and prognosis. J Clin Oncol 1994; 12: 1673- 84. 